\title{
Thermal Shock Properties of ZrB2-SiCp-Graphite and ZrB2-SiCp-AIN Ceramic Matrix Composite Material
}

\author{
Baoliang Liu ${ }^{*}, 1$, Jianguo Wang ${ }^{1}$, Guoqian Liu $^{2}$ \\ ${ }^{I}$ Department of Mathematics and Mechanics, Heilongjiang Institute of Science and Technology, Harbin 150027, P.R. \\ China \\ ${ }^{2}$ Research Laboratory on Composite Materials Harbin Institute of Technology, Harbin, 150001, P.R. China
}

\begin{abstract}
Thermal shock property of the material can be improved by improving fracture property of the material. In this paper, factors affecting fracture property have been studied and the effect of residual stress, microstructure, strain rate and stress concentration on fracture property of material has been analyzed, which may provide help for improving thermal shock property of material. Thermal shock stability for ZrB2-SiCp-Graphite(ZSG) and ZrB2-SiCp-AlN (ZSA) was investigated by water quenching test, which indicated that ZSC may provide more stable thermal shock properties. As shown by SEM of ZSA, surface cracks appeared after it was cooled from $1200^{\circ} \mathrm{C}$ due to thermal shock instability of the material. Residual flexural strength of ZSA was improved by crack healing after it was cooled from $1450^{\circ} \mathrm{C}$. However, no surface crack appeared for ZSG after water quenching test. This paper may provided a potential method for improving thermal shock stability of zirconium diboride ceramic matrix composites by introducing proper quantities of graphite.
\end{abstract}

Keywords: Ceramic-matrix composites; thermal shock, microstructures, mechanical properties, fracture property.

\section{INTRODUCTION}

As boride based ceramic matrix composite with high temperature resistance, eroded resistance, mechanical and chemical stability under high temperature, it becomes one of the most prospective materials for application in spaceflight, ultrasonic aircraft, and propulsion system of rocket. However, as brittleness of the material, catastrophic damage may be caused by thermal shock [1-8]. It is necessary to improve thermal shock properties of ceramic matrix composite, and characterize thermal shock properties of ceramic matrix composite properly $[9,10]$.

Zirconium diboride (ZrB2) has many intrinsic characteristics, such as high melting point, high hardness, good chemical inertness, and high wear resistance, which make it a promising candidate for high temperature structural materials [11-15]. However, thermal shock stability of pure $\mathrm{ZrB2}$ is very poor, which limits its application in re-entry space vehicles. Therefore, it is necessary to improve thermal shock resistance and thermal shock stability for $\mathrm{ZrB} 2$. It is a common way to improve thermal shock behavior by improving its mechanical properties (such as strength and toughness). Silicon carbide ( $\mathrm{SiC})$ particulates are common reinforcement for ceramic materials due to its performances of high strength, high elastic modulus under high temperature.

Many researchers [16-20] have investigated thermal shock behaviors of ceramic matrix composites in both theories and experiments for many years. However, few investigations were performed on thermal shock property of

*Address correspondence to this author at the Department of Mathematics and Mechanics, Heilongjiang Institute of Science and Technology, Harbin 150027, China; Tel: +86-0451-88036203; Fax: +86-0451-88036205;

E-mail: baoliangliu2008@163.com, lbzaaa@163.com ultra-high temperature ceramic composites, such as large size $\mathrm{ZrB} 2$ ceramic matrix composites.

In this work, large size materials were fabricated by hotpressing sintering: ZrB2-SiCp-Graphite (ZSG) and ZrB2$\mathrm{SiCp}-\mathrm{AlN}$ (ZSA). Mechanical properties and thermal shock behavior for the two materials were studied.

\section{EXPERIMENTAL}

The mean size of $\mathrm{ZrB} 2$ powder was about $2 \mu \mathrm{m}$, and the size of $\mathrm{SiC}$ particles was about $1 \sim 2 \mu \mathrm{m}$. The powder mixtures ( $\mathrm{ZrB} 2+20 \% \mathrm{SiC}+15 \%$ Graphite) were ball-milled for $10 \mathrm{~h}$ in a polyethylene bottle using $\mathrm{ZrO} 2$ balls and ethanol as grinding media. After mixing, the slurry was dried in a rotary evaporator. In order to sieve agglomeration formed during drying the slurry, the dried slurry was then screened using sieve with the mesh size $20 \mu \mathrm{m}$. The resulting powder mixtures were hot-pressed at $1900{ }^{\circ} \mathrm{C}$ for $1 \mathrm{~h}$ under a uniaxial load of $30 \mathrm{MPa}$ in $\mathrm{Ar}$ atmosphere. The same procedures were implemented for $\mathrm{ZrB} 2-20 \% \mathrm{SiC}-5 \% \mathrm{AlN}$ mixtures. Two kinds of materials with a same $\operatorname{size}(\varphi 80 \mathrm{~mm} \times 65 \mathrm{~mm})$ were fabricated.

Mechanical properties of the two materials were measured by using Instron 5848 MicroTester. Specimens were cut by linear cutting machine, which was a cutting machine for electric materials. Cutting process was dominated by electricity, which passed through electric wire whose diameter was $0.2 \mathrm{~mm}$. Residual flexural strength was tested in three-point flexural test on $3 \mathrm{~mm} \times 4 \mathrm{~mm} \times 36 \mathrm{~mm}$ bars, using $30 \mathrm{~mm}$ span and a crosshead speed of $0.5 \mathrm{~mm}$ min-1. Thermal conductivity was measured by Flash line 5000 (ANTER CORPORATION USA), and coefficient of thermal expansion was measured by UnithermTM 1252(ANTER CORPORATION USA). 
Electric muffle furnace was used as quenching test equipment. Meanwhile, boiling water was used as quenching medium. Temperature of heating and cooling systems of the quenching test apparatus for this experiment could be controlled within the limit of $\pm 10^{\circ} \mathrm{C}$. In each thermal shock test, a fresh specimen was heated for $10 \mathrm{~min}$ in the furnace and cooled for $5 \mathrm{~min}$ in the cooling bath to assure the temperature of the specimen uniform. After quenching test, specimens were dried and their residual strength values were measured using three-point flexural fixture with a span of $30 \mathrm{~mm}$ and a crosshead speed of $0.5 \mathrm{~mm}$ min- 1 with the temperature interval of $100^{\circ} \mathrm{C}$. Surfaces of specimens were observed by scanning electron microscopy.

\section{RESULTS AND DISCUSSION}

Fracture surface of the two materials are shown in Fig. (1). Fig. (1a) shows that fracture mode of ZSA is transgranular and intergranular mixed mode. And Fig. (1b) shows that flake shape graphite is observed in ZSG, which may dissipate much energy during crack propagating [21].

Fig. (2a) shows the thermal conductivities and coefficients of thermal expansion of the two materials at different temperatures. The thermal conductivities of ZSG are higher than that of ZSA nearly at all temperatures, while coefficients of thermal expansion of ZSG are relatively lower. Therefore, temperature gradient and thermal strain should be relatively smaller for ZSG during thermal shock than that for ZSA. All the reasons above may contribute to more stable thermal shock properties for ZSG, and it can be observed in Fig. (2b).

As shown in Fig. (2b), residual flexural strength of ZSA decreases abruptly at the quenching temperature of $n$ hed fromh additive of $\mathrm{AlN}$ a $1200^{\circ} \mathrm{C}$, and increases abruptly at the quenching temperature higher than $1200^{\circ} \mathrm{C}$. Although residual flexural strength of ZSG is much lower than that of ZSA, it varies little with quenching temperatures. At the quenching temperature of $1200^{\circ} \mathrm{C}$, average residual flexural strength of ZSA is almost the same as that of ZSG. However, the strengths of ZSA scatter in a much larger range than that of ZSG. Therefore, ZSG is safer used as aerospace material than ZSA if thermal shock stability is considered only.

Fig. (3a) shows the scanning electron microscopy of ZSA after it was cooled into boiling water from $1200^{\circ} \mathrm{C}$. The picture implied that, at the quenching temperature of $1200^{\circ} \mathrm{C}$, surface thermal stress induced by temperature gradient reached strength of the material. So, cracks were formed on the surface of specimen, which contributed to stress concentration at the cracks tip. Therefore, flexural strengths of the material after quenching test become lower at this quenching temperature. Furthermore, much more glass phase generated on the surface of specimen after it was heated to $1450^{\circ} \mathrm{C}$. As glass phase was liquid phase when temperature was higher than $800^{\circ} \mathrm{C}$, it filled up the surface cracks formed during quenching test from $1450^{\circ} \mathrm{C}$ to $800^{\circ} \mathrm{C}$ as shown by circle in Fig. $(\mathbf{3 b}, \mathbf{c})$. As a result, residual flexural strength at room temperature was improved.

Thermal shock stability is one of main requirements for the application of ultra high temperature ceramics (UHTC). This study clearly shows that it is a potential way for improving thermal shock stability of ZrB2 based UHTC by introducing proper quantities of graphite. (a) ZSA

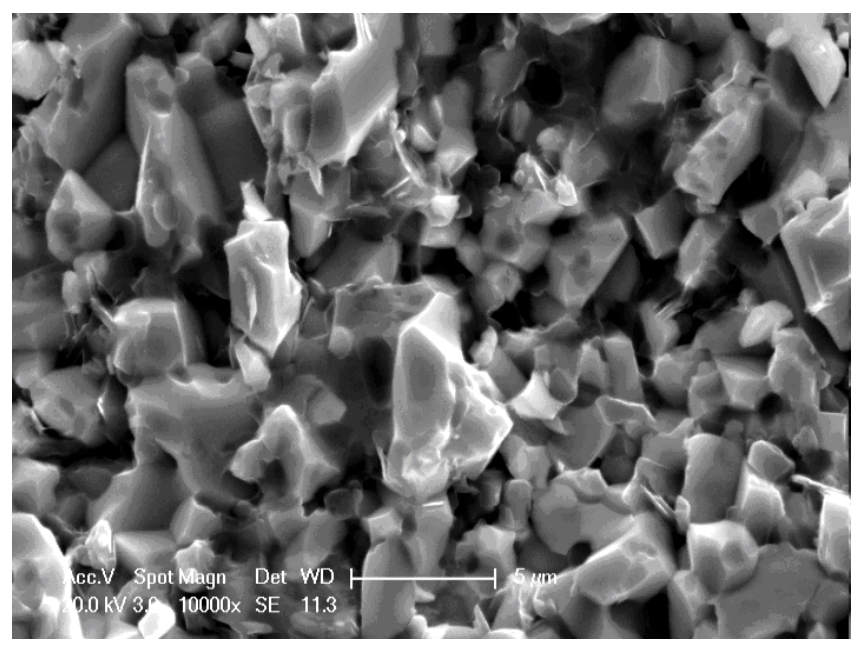

(b) ZSC

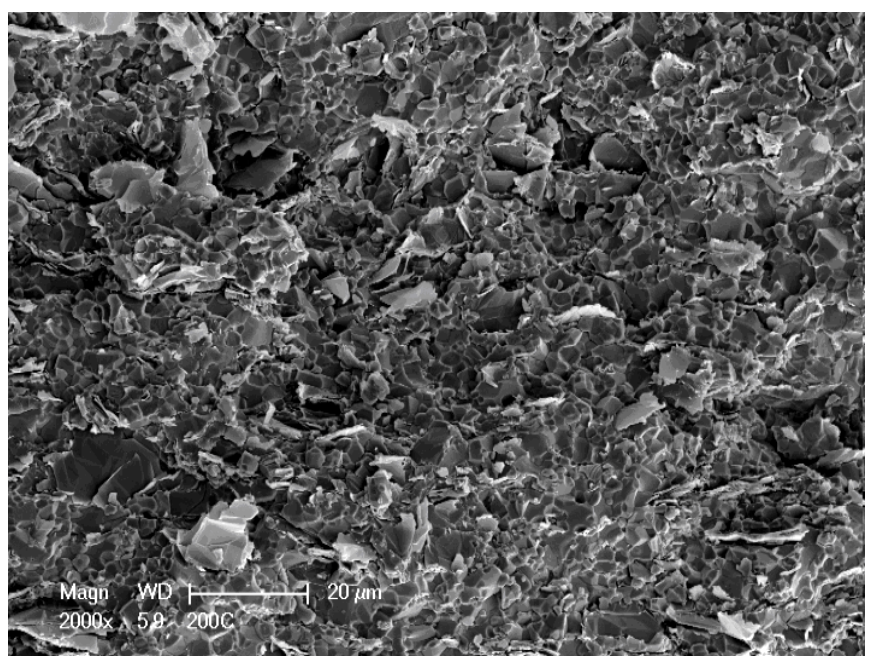

Fig. (1). Fracture surface pattern of (a) ZSA and (b) ZSC.

Thermal shock property of UHTC was investigated by quenching test, and two factors of microstructure and quenching environment affecting thermal shock property of UHTC were investigated. Result shows that as flake shape graphite can improve crack resistance of material, residual strength of the material with flake shape graphite is relatively higher. Material strength can be improved by decreasing grain size, so critical temperature difference was improved by decreasing grain size. However, limited by process, material strength distribution of UHTC with smaller grain size was large, and stability of mechanical property for the material was worse. Density of surface crack is not the main factor affecting residual strength, and crack depth is in a stable range after critical quenching difference, as a result, crack depth is the main factor. Heat transfer coefficients are different for UHTC with different quenching medium, so thermal shock properties of the same material are different when quenched into different medium. It was proved by finite element method that heat transfer coefficients and quenching temperature difference affect material failure by affecting surface heat transfer velocity. Namely, surface heat transfer velocity was the natural factor for controlling thermal shock failure. 
(a)

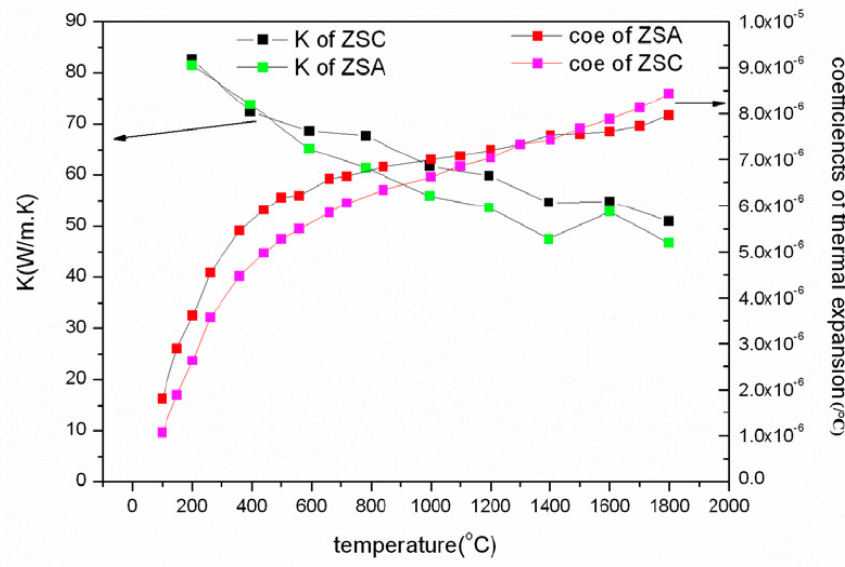

(b)

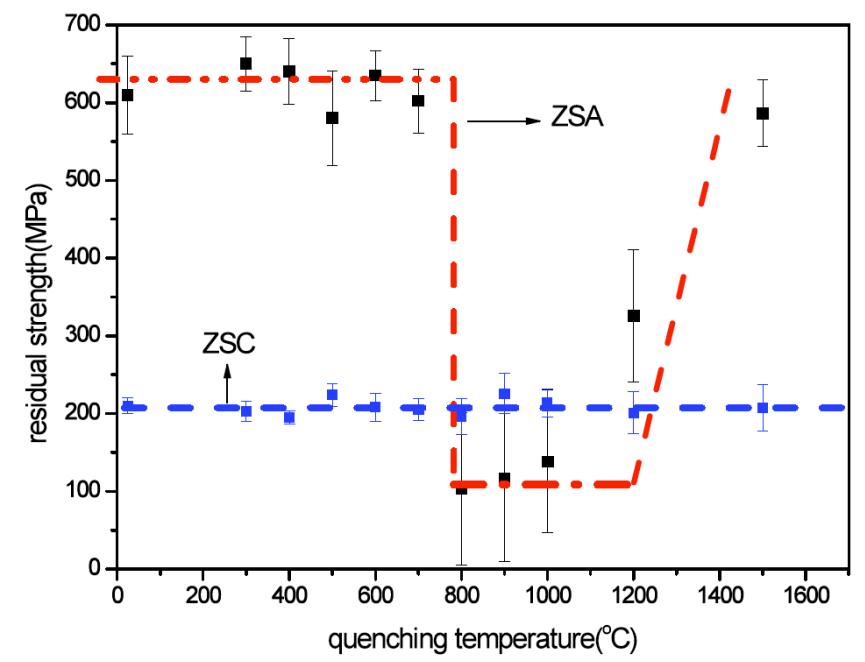

Fig. (2). (a) Thermal properties of the two materials; (b) Residual flexural strength of ZSC and ZSA after cooled into boiling water

\section{CONCLUSIONS}

Large size ceramic is needed for the future applications, and scale effect is one of dominating factors for the properties of the sintered material. In other words, hot pressing sintering scale becomes larger, density and mechanical properties become lower for the ceramic. In order to investigate the properties of large scale ceramic, large scale ZrB2 ceramic matrix composites (ZSC and ZSA) were fabricated by hot-pressing sintering. Thermal conductivities of ZSC are relatively higher, which make it a relatively lower temperature gradient during thermal shock. In addition, coefficients of thermal expansion for ZSC are relatively lower nearly at all measured temperatures. Therefore, thermal shock stability of ZSC is higher than that of ZSA, although mechanical property of ZSC is relatively lower.

The results in the paper clearly shows that it is a potential way to improve thermal shock stability by improving thermal conductivity, and reducing coefficient of thermal expansion, except improving the mechanical properties of materials. (a) $1200^{\circ} \mathrm{C}$

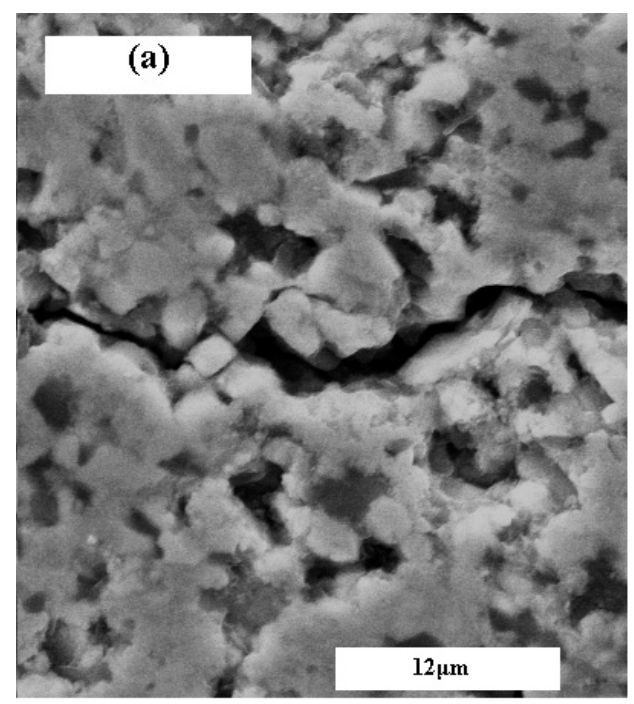

(b) $1450^{\circ} \mathrm{C}$

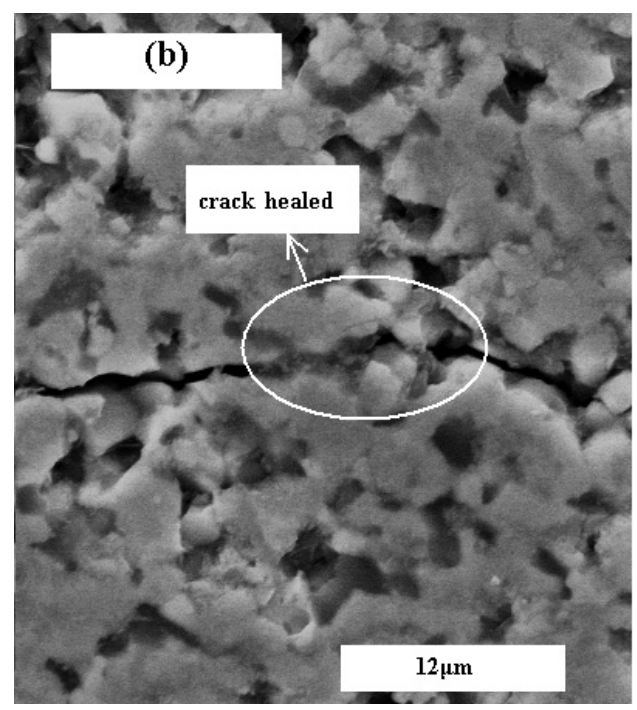

(c) $1450^{\circ} \mathrm{C}$

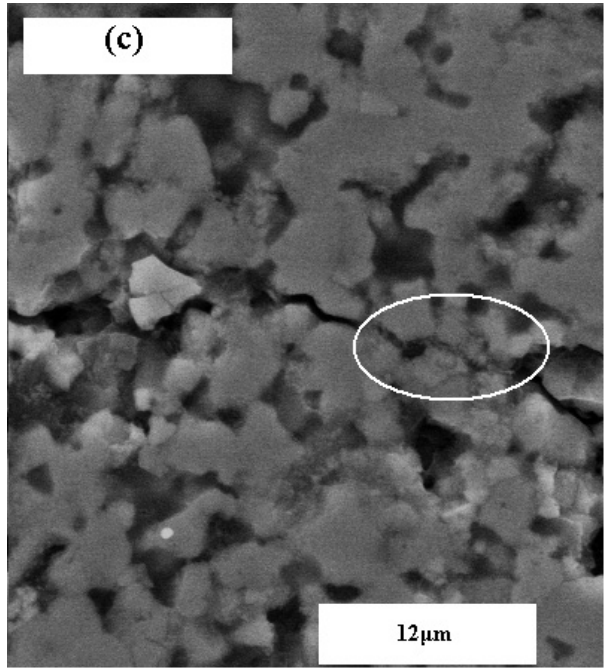

Fig. (3). Surface pattern of ZSA cooled from (a) $1200^{\circ} \mathrm{C}$ (b) $1450^{\circ} \mathrm{C}$ (c) $1450^{\circ} \mathrm{C}$. 


\section{REFERENCES}

[1] Upadhya K, Yang JM, Hoffmann WP. Materials for ultra high temperature structural applications. Am Ceram Soc Bull 1997; 76(12): 51-6.

[2] Fahrenholtz WG, Hilmas GE, Talmy IG, Zaykoski JA. Refractory diborides of zirconium and hafnium. J Am Ceram Soc 2007; 90(5): 1347-64.

[3] Clougherty EV, Pober RL, Kaufman L. Synthesis of Oxidation Resistant Metal Diboride Composites. Trans Met Soc AIME 1968; 242: 1077-82.

[4] Zhang LT, Cheng LF. Progress in research work of new CMC-SiC [J]. AMT 2003; 1: 24-32.

[5] Brown AS. Hypersonic designs with a sharp edge. Aerospace Am 1997; 35(9): 20-21.

[6] Kuwabara K. Some characteristics and applications of $\mathrm{ZrB} 2$ ceramics. Bull Ceram Soc Jpn 2002; 37(4): 267-71.

[7] Norasetthekul S, Eubank PT, Bradley WL, Bozkurt B, Stucker B. Use of zirconium diboride-copper as an electrode in plasma applications. J Mater Sci 1999; 34(6): 1261-70.

[8] Loehman R, Corral E, Dumm HP, Kotula P, Tandon R. Ultra High Temperature Ceramics for Hypersonic Vehicle Applications, Sandia National Laboratories Report, SAND 2006; 2006-2925.

[9] Telle R, Sigl LS, Takagi K. Boride-based hard materials. In: Riedel $\mathrm{R}$, Ed. Handbook of Ceramic Hard Materials, Wiley-VCH, Weinheim 2000; vol. 2: pp. 802-945.

[10] Levine SR, Opila EJ, Halbig MC, Kiser JD, Singh M, Salem JA. Evaluation of ultra-high temperature ceramics for aeropropulsion use. J Eur Ceram Soc 2002; 22: 2757-67.

[11] Stanley RL, Elizabeth JO, Michael CH, James DK, Mrityunjay S, Jonathan AS. Evaluation of ultra-high temperature ceramics foraeropropulsion use. J Eur Ceram Soc 2002; 222: 757-67.
[12] Fr'ed'eric M, Raffaele S. Stability of ultra-high-temperature ZrB2$\mathrm{SiC}$ ceramics under simulated atmospheric re-entry conditions. J Eur Ceram Soc 2007; 27: 4797-805.

[13] Chamberlain AL, Fahrenholtz WG, Hilmas GE, Ellerby DT. High Strength Zirconium Diboride-Based Ceramics. J Am Ceram Soc 2004; 87: 1170-2.

[14] Zhang G-J, Zhen-Yan D, Naoki K, Jian-Feng Y, Tatsuki O. Reactive hot pressing of $\mathrm{ZrB} 2-\mathrm{SiC}$ composites. J Am Ceram Soc 2000; 83: 2330-2.

[15] Xing HZ, Ping H, Jie CH, Lin X, Song HM. The addition of lanthanum hexaboride to zirconium diboride for improved oxidation resistance. Scr Mater 2007; 57: 1036-9.

[16] Zhi-Hua Y, De-Chang J, Yu Z, Qing-Chang M, Peng-Yuan S, Cheng-Bin $\mathrm{S}$. Thermal shock resistance of in situ formed $\mathrm{SiC}-\mathrm{BN}$ composites. Mater Chem Phys 2008; 107: 476-9.

[17] Kalantar M, Fantozzi G. Thermo-mechanical properties of ceramics: Resistance to initiation and propagation of crack in high temperature. Mater Sci Eng A 2008; 472: 273-80.

[18] Liya S, Mingjian L, Xiaozhen L, Bo L. Thermal shock resistance of the porous $\mathrm{A} 12 \mathrm{O} 3 / \mathrm{ZrO} 2$ ceramics prepared by gelcasting. Mater Res Bull 2007; 42: 2048-56.

[19] Sands CM, Henderson RJ, Chandler HW. A three dimensional computational model of the mechanical response of a dual-phase ceramic. Comp Mater Sci 2007; 39: 862-70.

[20] Joong HL, Sung EP, Hyung JL, Hong LL. Thermal shock behaviour of alumina ceramics by ball-on-3-ball test. Materials Letter 2002; 56: 1022-9.

[21] Songhe M, Guoqian L, Jing A, Shilong S. Effect of different additives on microstructure and crack resistance for an ultra high temperature ceramic. International journal of refractory metal and ceramic 2009; 27: 813-6.

(C) Liu et al.; Licensee Bentham Open.

This is an open access article licensed under the terms of the Creative Commons Attribution Non-Commercial License (http://creativecommons.org/licenses/ by$\mathrm{nc} / 3.0 /$ ) which permits unrestricted, non-commercial use, distribution and reproduction in any medium, provided the work is properly cited. 